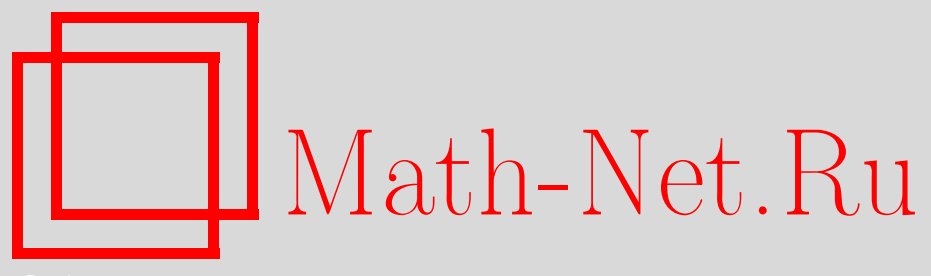

С. Я. Старцев, Метод каскадного интегрирования Лапласа для линейных гиперболических систем уравнений, Матем. заметки, 2008, том 83, выпуск 1, 107-118

DOI: https://doi.org/10.4213/mzm4338

Использование Общероссийского математического портала Math-Net.Ru подразумевает, что вы прочитали и согласны с пользовательским соглашением http://www.mathnet.ru/rus/agreement

Параметры загрузки:

IP : 3.95 .254 .165

26 апреля 2023 г., 15:34:40

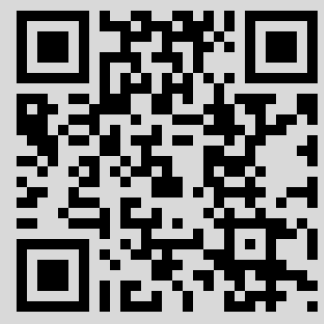




\section{Математические заметки}

Том 83 выпуск 1 январь 2008

УДК 517.956.32

\section{Метод каскадного интегрирования Лапласа для линейных гиперболических систем уравнений}

\section{С. Я. Старцев}

Предложено обобщение каскадного метода интегрирования Лапласа на случай линейных гиперболических систем уравнений. На основе этого доказано, что система уравнений с обращающимся в нуль произведением инвариантов Лапласа обладает полным набором решений, зависящих от произвольных функций.

Библиография: 9 названий.

1. Введение: каскадное интегрирование скалярных уравнений. Рассмотрим систему линейных уравнений вида

$$
l(u) \equiv u_{x y}+a(x, y) u_{x}+b(x, y) u_{y}+c(x, y) u=0,
$$

где через $a, b$ и $c$ обозначены матрицы размера $n \times n$, а $u$ является $n$-мерным вектором. В скалярном случае (при $n=1$ ) одним из классических методов точного интегрирования уравнений вида (1) является каскадный метод интегрирования Лапласа (см., например, [1]-[3]). Однако для систем уравнений (т.е. когда $n>1$ ) такого метода не имеется и целью настоящей работы будет обобщение каскадного метода интегрирования на случай произвольного $n$.

В качестве введения положим $n=1$ и опишем, как выглядит интересующий нас метод для скалярных уравнений. Несколько необычный вид некоторых формул, используемых в ходе этого изложения, объясняется желанием записать эти формулы таким образом, чтобы они оставались пригодными и для систем уравнений.

Нетрудно видеть, что дифференциальный оператор $l$, задающий уравнение (1), может быть записан в виде

$$
l=\left(\frac{\partial}{\partial x}+b\right) \circ\left(\frac{\partial}{\partial y}+a\right)-H_{1},
$$

где символом о обозначена операция композиции операторов. Остаток $H_{1}$ от деления оператора $l$ на оператор $\partial / \partial y+a$ назовем $у$-инвариантом Лапласа уравнения (1). Легко проверить, что $H_{1}=a_{x}+b a-c$. Если $H_{1}=0$, то решение уравнения (1) может быть найдено путем последовательного интегрирования двух обыкновенных

Работа выполнена при поддержке Российского фонда фундаментальных исследований (грант № 05-01-00775-a).

(C) С.Я. Старцев, 2008 
дифференциальных уравнений с параметром. Если же $H_{1} \neq 0$, то можно построить дифференциальную подстановку, переводящую в решения уравнения (1) решения некоторого другого уравнения такого же вида. Суть каскадного метода интегрирования состоит в том, чтобы связать исходное уравнение цепочкой таких подстановок с уравнением, у которого $y$-инвариант Лапласа будет равен нулю.

Для построения упомянутой дифференциальной подстановки найдем функцию $a_{1}(x, y)$ такую, что выполнено операторное соотношение

$$
\left(\frac{\partial}{\partial y}+a_{1}\right) \circ H_{1}=H_{1} \circ\left(\frac{\partial}{\partial y}+a\right) .
$$

Если $H_{1} \neq 0$, то это соотношение однозначно определяет $a_{1}=a-\left(H_{1}\right)_{y} / H_{1}$ и верна формула

$$
\left(\frac{\partial}{\partial y}+a\right) \circ \frac{1}{H_{1}}=\frac{1}{H_{1}} \circ\left(\frac{\partial}{\partial y}+a_{1}\right) .
$$

Пользуясь этой формулой и учитывая (2), нетрудно проверить, что оператор

$$
l_{1}=\left(\frac{\partial}{\partial y}+a_{1}\right) \circ\left(\frac{\partial}{\partial x}+b\right)-H_{1}
$$

связан с оператором $l$ соотношением

$$
l \circ \frac{1}{H_{1}} \circ\left(\frac{\partial}{\partial x}+b\right)=\left(\frac{\partial}{\partial x}+b\right) \circ \frac{1}{H_{1}} \circ l_{1} .
$$

Последнее равенство означает, что оператор $\left(H_{1}\right)^{-1}(\partial / \partial x+b)$ переводит ядро оператоpa $l_{1}$ в ядро оператора $l$, т.е. дифференциальная подстановка $u=\left(H_{1}\right)^{-1}\left(v_{x}+b(x, y) v\right)$, называемая преобразованием Лапласа, переводит решения уравнения $l_{1}(v)=0$ в решения уравнения (1).

Найдем $y$-инвариант Лапласа $H_{2}$ уравнения $l_{1}(v)=0$. По определению $H_{2}$ есть остаток от деления оператора $l_{1}$ на оператор $\partial / \partial y+a_{1}$, т.е.

$$
l_{1}=\left(\frac{\partial}{\partial x}+b\right) \circ\left(\frac{\partial}{\partial y}+a_{1}\right)-H_{2} .
$$

Сравнив последнюю формулу с (4), получаем

$$
H_{2}=H_{1}+\left[\frac{\partial}{\partial x}+b, \frac{\partial}{\partial y}+a_{1}\right],
$$

где через $[f, g]$ обозначен коммутатор $f \circ g-g \circ f$ операторов $f$ и $g$. Вычислив коммутатор, получаем $H_{2}=H_{1}+\left(a_{1}\right)_{x}-b_{y}$. Если $H_{2} \neq 0$, то мы можем повторить рассуждения из предыдущего абзаца применительно к уравнению $l_{1}(v)=0$ и однозначно определить дифференциальный оператор $l_{2}$ такой, что дифференциальная подстановка $v=\left(H_{2}\right)^{-1}\left(w_{x}+b(x, y) w\right)$ будет переводить решения уравнения $l_{2}(w)=0$ в решения уравнения $l_{1}(v)=0$.

Многократно повторяя вышеприведенные рассуждения, мы можем построить последовательность дифференциальных операторов

$$
l_{i}=\left(\frac{\partial}{\partial x}+b\right) \circ\left(\frac{\partial}{\partial y}+a_{i}\right)-H_{i+1}
$$


такую, что оператор $\left(H_{i}\right)^{-1}(\partial / \partial x+b)$ переводит ядро оператора $l_{i}$ в ядро оператоpa $l_{i-1}$. Функции $a_{i}$ и $H_{i+1}$ при этом будут определяться по рекуррентным формулам

$$
\begin{gathered}
\left(\frac{\partial}{\partial y}+a_{i}\right) \circ H_{i}=H_{i} \circ\left(\frac{\partial}{\partial y}+a_{i-1}\right), \\
H_{i+1}=H_{i}+\left[\frac{\partial}{\partial x}+b, \frac{\partial}{\partial y}+a_{i}\right] .
\end{gathered}
$$

Будем продолжать эту последовательность до тех пор, пока очередной $y$-инвариант Лапласа $H_{i+1}$ не окажется равным нулю, и потому можем считать, что $H_{j} \neq 0$ для всех $j \leqslant i$. Заметим, что в качестве определяющих соотношений для $a_{i}$ мы можем взять операторные равенства

$$
\left(\frac{\partial}{\partial y}+a_{i}\right) \circ H_{i} H_{i-1} \cdots H_{1}=H_{i} H_{i-1} \cdots H_{1} \circ\left(\frac{\partial}{\partial y}+a\right),
$$

так как и формулы (6), и формулы (8) дают нам

$$
a_{i}=a-\frac{\left(H_{i} H_{i-1} \cdots H_{1}\right)_{y}}{H_{i} H_{i-1} \cdots H_{1}}
$$

для всех $i$ таких, что $H_{i} H_{i-1} \cdots H_{1} \neq 0$. Поскольку $a_{i}$ определены однозначно, то и $y$-инварианты Лапласа $H_{i+1}$ определены однозначно вплоть до момента, когда очередной инвариант Лапласа обратиться в нуль.

Предположим теперь, что найдется такое $m$, что $H_{m+1}=0$. Тогда

$$
l_{m}=\left(\frac{\partial}{\partial x}+b\right) \circ\left(\frac{\partial}{\partial y}+a-\frac{\left(H_{m} H_{m-1} \cdots H_{1}\right)_{y}}{H_{m} H_{m-1} \cdots H_{1}}\right)
$$

и $w=H_{m} H_{m-1} \cdots H_{1} \gamma(x, y) f(x)$, где $\gamma_{y}+a \gamma=0$, является решением уравнения $l_{m}(w)=0$ для любой функции $f(x)$. Воспользовавшись преобразованиями Лапласа, из этого решения получим следующее решение исходного уравнения (1):

$$
u=\frac{1}{H_{1}} \circ\left(\frac{\partial}{\partial x}+b\right) \circ \cdots \circ \frac{1}{H_{m}} \circ\left(\frac{\partial}{\partial x}+b\right)\left(H_{m} H_{m-1} \cdots H_{1} \gamma(x, y) f(x)\right) .
$$

Заметим, что выбрав функции $B_{i}(x, y)$ так, чтобы выполнялись операторные соотношения

$$
\left(\frac{\partial}{\partial x}+b\right) \circ H_{i} H_{i-1} \cdots H_{1}=H_{i} H_{i-1} \cdots H_{1} \circ\left(\frac{\partial}{\partial x}+B_{i}\right),
$$

нетрудно переписать полученную нами формулу для решений уравнения (1) в виде

$$
u=\left(\frac{\partial}{\partial x}+B_{1}\right) \circ\left(\frac{\partial}{\partial x}+B_{2}\right) \circ \cdots \circ\left(\frac{\partial}{\partial x}+B_{m}\right)(\gamma(x, y) f(x)) .
$$

Ясно, что мы можем удовлетворить соотношениям (9), положив

$$
B_{i}=b+\frac{\left(H_{i} H_{i-1} \cdots H_{1}\right)_{x}}{H_{i} H_{i-1} \cdots H_{1}} .
$$


Раскрыв скобки в (10), найденное решение уравнения (1) можем записать также еще и в виде

$$
u=\sum_{i=0}^{m} S_{i}(x, y) \frac{d^{i} f(x)}{d x^{i}} .
$$

Для более компактной записи формул здесь и далее будем придерживаться того соглашения, что $d^{0} f / d x^{0}=f$.

Таким образом, применение каскадного метода интегрирования Лапласа в скалярном случае дает нам следующее хорошо известное

УтВеРЖДЕНИЕ 1. Обозначим через $H_{i}$ последователъность функиий, заданную рекуррентными формулами (7), (8) и первым членом $H_{1}=a_{x}+b a-c$. Пусть найдется $m$ такое, что $H_{m+1}=0$. Тогда найдутся функиии $S_{i}(x, y)$ такие, что $S_{m} \neq 0$ и (11) является решением скалярного $(n=1)$ уравнения (1) для любой функиии $f(x)$.

Ясно, что таким же образом мы можем определить последовательность $x$-инвариантов Лапласа и доказать для нее утверждение, аналогичное утверждению 1, для этого достаточно в вышеприведенных рассуждениях поменять местами $x$ и $y$, а также $a$ и $b$. Пользуясь симметрией формулы (1) относительно перестановок $x \leftrightarrow$ $y, a \leftrightarrow b$, в настоящей статье будем приводить лишь одно из двух "симметричных" утверждений и определений.

Заметим, что не любое уравнение (1) может быть проинтегрировано с помощью каскадного метода Лапласа - условием применимости этого метода в скалярном случае является выполнение равенства $H_{i}=0$ при некотором $i$. В следующей части статьи мы покажем, какие условия следует считать достаточно естественными для применимости этого метода в случае систем и на основе этого сформулируем для систем теорему, аналогичную утверждению 1.

2. Определения, известные факты и вспомогательные утверждения. Отсюда и далее будем работать с системами линейных уравнений вида (1), т.е. будем считать $n$ произвольным. При этом, однако, мы будем ссылаться на те из приведенных выше формул, которые остаются пригодными и для систем, т.е. не требуют переписывания при замене в них скалярных функций на матрицы с теми же обозначениями. Не оговаривая это каждый раз особо, далее будем считать все вектора $n$-мерными, а матрицы - имеющими размер $n \times n$. Если не указано иного, компоненты всех векторов и матриц следует считать функциями от $x$ и $y$.

Так же, как и в скалярном случае, инвариант Лапласа $H_{1}$ определим по формуле (2) как остаток от деления оператора $l$ на оператор $\partial / \partial y+a$. Так как $H_{1}=$ $a_{x}+b a-c$ теперь является матрицей, которая, вообе говоря, может быть вырожденной, формула (5) становится непригодной и прямого аналога преобразования Лапласа в случае систем не имеется. Однако можем попробовать построить последовательность матриц $H_{i}$, определяя, по аналогии со скалярным случаем, матрицы $a_{i}$ из формулы (8) и вычисляя $H_{i+1}$ по формуле $(7)$. Вычислив коммутатор в $(7)$ и обозначив через $Y_{i}$ произведение матриц $H_{i} H_{i-1} \cdots H_{1}$, приходим к следующему определению, предложенному в работе [4]. 
ОПРЕДЕЛЕНИЕ 1. Обобщенными у-инвариантами Лапласа системы (1) называются матрицы $Y_{i}$, определяемые по рекуррентным формулам

$$
\begin{gathered}
Y_{1}=H_{1}=a_{x}+b a-c, \quad Y_{i+1}=H_{i+1} Y_{i}, \\
H_{i+1}=\frac{\partial a_{i}}{\partial x}+\left[b, a_{i}\right]-\frac{\partial b}{\partial y}+H_{i},
\end{gathered}
$$

где матрицы $a_{i}$ выбраны так, чтобы выполнялись операторные соотношения

$$
\left(\frac{\partial}{\partial y}+a_{i}\right) \circ Y_{i}=Y_{i} \circ\left(\frac{\partial}{\partial y}+a\right) .
$$

Формула (14) равносильна следующему матричному уравнению:

$$
\frac{\partial Y_{i}}{\partial y}-Y_{i} a+a_{i} Y_{i}=0
$$

которое в случае $\operatorname{det}\left(Y_{i}\right)=0$ может не иметь решения $a_{i}$. Поэтому существование последовательности инвариантов Лапласа нужной длины не гарантировано для какой угодно системы вида (1). И если в скалярном случае условием применимости метода каскадного интегрирования являлось обращение в нуль инварианта Лапласа $Y_{m+1}$ при некотором $m$, то в случае систем к этому условию приходится добавлять еще и предположение о разрешимости соотношений (14) для всех $i \leqslant m$.

Кроме того, для вырожденного $Y_{i}$ матрица $a_{i}$ (если она существует) определяется из (15) неоднозначно, а стало быть и последовательность обобщенных инвариантов также определена, вообще говоря, неоднозначно. Исходя из нежелательности работы с некорректно определенными объектами, естественно потребовать от системы (1), чтобы ее инварианты Лапласа $Y_{i}$ не только существовали, но и не зависели от выбора решений уравнений (15). Из работ [4], [5] известно следующее

УтвеРжДЕНИЕ 2. Обобщенный инвариант Лапласа $Y_{k}$ системы (1) не зависит от выбора матрии, $a_{i}$ тогда и только тогда, когда для всех $i<k$ найдутся матрицы $B_{i}$, удовлетворяющие уравнениям

$$
\frac{\partial Y_{i}}{\partial x}+b Y_{i}-Y_{i} B_{i}=0
$$

Нетрудно проверить, что уравнение (16) равносильно операторному равенству

$$
\left(\frac{\partial}{\partial x}+b\right) \circ Y_{i}=Y_{i} \circ\left(\frac{\partial}{\partial x}+B_{i}\right) .
$$

Отметим, что функции $B_{i}$ в формуле $(10)$ для решений скалярного уравнения задаются как раз уравнением (17). Это совпадение можно рассматривать как дополнительный аргумент в пользу разумности предположения о единственности обобщенных инвариантов Лапласа системы (1).

Целью настоящей статьи является доказательство следующего аналога утверждения 1.

ТЕОРема 1. Пусть для системы (1) для всех $i \leqslant m$ найдутся матрицы $a_{i}$ и $B_{i}$ такие, что выполнены соотношения (14) и (17), где матрицы $Y_{i}$ вычислены по 
формулам (12), (13), и пусть $Y_{m+1}=0$. Тогда найдутся матрицы $S_{i}(x, y)$ такие, что

$$
u=\sum_{i=0}^{m} S_{i}(x, y) \frac{d^{i} f(x)}{d x^{i}}
$$

для любого вектора $f(x)$ является решением системы (1) $u \operatorname{det}\left(S_{m}\right) \neq 0$.

Другими словами, в теореме 1 предполагаем, что обобщенные инварианты Лапласа системы (1) существуют и определены однозначно ${ }^{1}$. Можно доказать, что при этих предположениях условие $Y_{m+1}=0$ является необходимым для существования решения вида (18) (cp. [5], [6]), однако такое доказательство выходит за рамки темы настоящей статьи. Заметим, что в работе [7] ранее уже был предложен алгоритм построения решений вида (18), но он требует выполнения условия значительно более жесткого, чем условие $Y_{m+1}=0$.

Для доказательства теоремы нам потребуются следующие результаты.

УтВеРЖДЕНИЕ 3. Матрица $a_{i}$ такая, что выполнено соотношение (14), существует тогда и только тогда, когда в ядре $Y_{i}$ можно выбрать базис из векторов $e_{j}$ maкux, что $\partial e_{j} / \partial y+a e_{j}=0$.

УТВЕРЖДЕНИЕ 4. Пусть для всех $j \leqslant m$ существуют обобщенные инварианты Лапласа $Y_{j}$ системы (1) и для всех $i<m$ найдутся матрицы $B_{i}$, удовлетворяющие уравнениям (17). Тогда для всех $i<m$ верна формула $Y_{i+1}=Y_{i} \widehat{H}_{i+1}$, где

$$
\widehat{H}_{1}=H_{1}, \quad \widehat{H}_{i+1}=\widehat{H}_{i}+\left[\frac{\partial}{\partial x}+B_{i}, \frac{\partial}{\partial y}+a\right] .
$$

Утверждения 3 и 4 были доказаны в работах [7] и [8] соответственно.

Мы будем стремиться провести доказательство теоремы 1 так, чтобы из него можно было легко извлечь способ построения решения (18). При практическом нахождении этого решения удобно сначала обратимыми преобразованиями привести систему (1) к некому каноническому виду. Для обоснования корректности этого действия следует доказать

УТВЕРЖДЕНИЕ 5. Пусть условия теоремы 1 выполнены для системы (1). Тогда система уравнений

$$
\left(\frac{\partial}{\partial x}+\widetilde{b}\right)\left(\frac{\partial}{\partial y}+\widetilde{a}\right) \widetilde{u}-\widetilde{H}_{1} \widetilde{u}=0,
$$

полученная из системы (1) заменой переменных $u=\gamma(x, y) \widetilde{u}$, где $\gamma$ - невырожденная матрица, также будет удовлетворять всем условиям теоремы.

ДокАзАтЕльство. Прямым вычислением получаем

$$
\begin{array}{lll}
\widetilde{a}=\gamma^{-1}\left(a \gamma+\gamma_{y}\right), & \left(\frac{\partial}{\partial y}+\widetilde{a}\right)=\gamma^{-1} \circ\left(\frac{\partial}{\partial y}+a\right) \circ \gamma, \\
\widetilde{b}=\gamma^{-1}\left(b \gamma+\gamma_{x}\right), & \left(\frac{\partial}{\partial x}+\widetilde{b}\right)=\gamma^{-1} \circ\left(\frac{\partial}{\partial x}+b\right) \circ \gamma
\end{array}
$$

\footnotetext{
ЭЭтим предположением не накладываем на систему никаких ограничений кроме тех, которые выполнены в скалярном случае, так как инварианты Лапласа скалярного уравнения всегда существуют и определены однозначно.
} 
и $\widetilde{H}_{1}=\gamma^{-1} H_{1} \gamma$. Стартуя с этих формул, индукцией по $i$ нетрудно доказать, что $\widetilde{H}_{i}=\gamma^{-1} H_{i} \gamma, \widetilde{Y}_{i}=\gamma^{-1} Y_{i} \gamma$ и условия теоремы выполнены для преобразованной системы. Действительно, из (14) следует

$$
\gamma^{-1} \circ\left(\frac{\partial}{\partial y}+a_{i}\right) \circ \gamma \gamma^{-1} Y_{i} \gamma=\gamma^{-1} Y_{i} \gamma \gamma^{-1} \circ\left(\frac{\partial}{\partial y}+a\right) \circ \gamma
$$

В силу предположения индукции $\widetilde{Y}_{i}=\gamma^{-1} Y_{i} \gamma$ последняя формула означает, что для $\widetilde{a}_{i}=\gamma^{-1}\left(a_{i} \gamma+\gamma_{y}\right)$ выполнено соотношение

$$
\left(\frac{\partial}{\partial y}+\widetilde{a}_{i}\right) \circ \widetilde{Y}_{i}=\widetilde{Y}_{i} \circ\left(\frac{\partial}{\partial y}+\widetilde{a}\right) .
$$

Подставив в формулу (13) предположение индукции $\widetilde{H}_{i}=\gamma^{-1} H_{i} \gamma$ и найденное значение $\widetilde{a}_{i}$, получаем

$$
\begin{aligned}
\widetilde{H}_{i+1} & =\frac{\partial \widetilde{a}_{i}}{\partial x}+\left[\widetilde{b}, \widetilde{a}_{i}\right]-\frac{\partial \widetilde{b}}{\partial y}+\widetilde{H}_{i}=\left[\frac{\partial}{\partial x}+\widetilde{b}, \frac{\partial}{\partial y}+\widetilde{a}_{i}\right]+\gamma^{-1} H_{i} \gamma \\
& =\left[\gamma^{-1} \circ\left(\frac{\partial}{\partial x}+b\right) \circ \gamma, \gamma^{-1} \circ\left(\frac{\partial}{\partial y}+a_{i}\right) \circ \gamma\right]+\gamma^{-1} H_{i} \gamma=\gamma^{-1} H_{i+1} \gamma
\end{aligned}
$$

и $\widetilde{Y}_{i+1}=\widetilde{H}_{i+1} \widetilde{Y}_{i}=\left(\gamma^{-1} H_{i+1} \gamma\right)\left(\gamma^{-1} Y_{i} \gamma\right)=\gamma^{-1} Y_{i+1} \gamma$. Аналогичные рассуждения приводят к тому, что $\widetilde{B}_{i}=\gamma^{-1}\left(B_{i} \gamma+\gamma_{x}\right)$ удовлетворяет равенству

$$
\left(\frac{\partial}{\partial x}+\widetilde{b}\right) \circ \widetilde{Y}_{i}=\tilde{Y}_{i} \circ\left(\frac{\partial}{\partial x}+\widetilde{B}_{i}\right) .
$$

Утверждение 5 доказано.

\section{3. Метод каскадного интегрирования.}

ДокАЗАТЕЛЬСТво тЕОРЕмы 1. Учитывая утверждение 5, без нарушения общности можем считать, что $a=0$, так как сделав в системе (1) замену переменных $u=\gamma(x, y) \widetilde{u}$, где $\gamma_{y}+a \gamma=0$, всегда можем обнулить матрицу $a$.

Обозначим

$$
L_{i}=\left(\frac{\partial}{\partial x}+b\right) \circ Y_{i} \circ \frac{\partial}{\partial y}-Y_{i+1} .
$$

Заметим, что считая $Y_{0}$ равным единичной матрице и учитывая равенство $Y_{1}=H_{1}$, получаем $L_{0}=l$. Для дальнейших рассуждений нам также потребуются дифференциальные операторы

$$
\widehat{l}_{i}=\left(\frac{\partial}{\partial x}+B_{i}\right) \circ \frac{\partial}{\partial y}-\widehat{H}_{i+1}
$$

где $B_{i}$ и $\widehat{H}_{i}$ находятся по формулам (17) и (19), соответственно. Учитывая (19), оператор $\widehat{l_{i}}$ можем также записать в виде

$$
\widehat{l}_{i}=\frac{\partial}{\partial y} \circ\left(\frac{\partial}{\partial x}+B_{i}\right)-\widehat{H}_{i}=0 .
$$


Предположим, что существуют матрицы $\gamma_{j}$ такие, что

$$
u_{i}=\frac{d^{p} f}{d x^{p}}+\sum_{j=0}^{p-1} \gamma_{j}(x, y) \frac{d^{j} f}{d x^{j}}
$$

является решением системы уравнений $L_{i}(u)=0$ для любого вектора $f(x)$. Покажем, что тогда найдутся матрицы $\xi_{j}$ такие, что для дифференциального оператора

$$
R_{i}=\sum_{j=0}^{p} \xi_{j}(x, y) \frac{d^{j}}{d x^{j}}
$$

выполнены равенства

$$
\frac{\partial R_{i}(f)}{\partial y}=\widehat{l}_{i}\left(u_{i}\right), \quad Y_{i} R_{i}(f)=0 .
$$

Нетрудно проверить, что

$$
\widehat{l}_{i}\left(u_{i}\right)=\sum_{j=0}^{p} \eta_{j}(x, y) \frac{d^{j} f}{d x^{j}},
$$

где $\eta_{j}$ - некоторые матрицы. В силу (17) и утверждения 4 выполнены соотношения $L_{i}=Y_{i} \widehat{l}_{i}$ и, следовательно, из $L_{i}\left(u_{i}\right)=0$ вытекает $\widehat{l}_{i}\left(u_{i}\right) \in \operatorname{ker} Y_{i}$. Таким образом, $Y_{i} \eta_{j}=0$ для всех $j \leqslant p$. Выбрав матрицы $\xi_{j}$ в операторе $R_{i}$ так, что $\partial \xi_{j} / \partial y=$ $\eta_{j}$, выполним первое из условий (23). Покажем теперь, что мы можем при этом удовлетворить и второму из условий (23), выбрав константы интегрирования так, чтобы столбцы матриц $\xi_{j}$ также лежали в ядре $Y_{i}$. Для этого воспользуемся тем, что согласно утверждению 3 в $\operatorname{ker} Y_{i}$ можно выбрать базис $e_{1}(x), \ldots, e_{k}(x)$ из векторов, зависящих только от $x$. Произвольный вектор $r(x, y)$ из ядра $Y_{i}$ разлагается по этому базису: $r(x, y)=\sum_{j=1}^{k} e_{j}(x) c_{j}(x, y)$. Тогда

$$
\int r d y=g(x)+\sum_{j=1}^{k} e_{j}(x) \int c_{j}(x, y) d y
$$

где $g(x)$ - вектор, составленный из констант интегрирования. Выбрав $g=0$, получим $Y_{i} \int r d y=0$.

Пусть теперь нам известно решение $u_{i}$ вида $(22)$ для системы $L_{i}(u)=0$ и соответствующий ему оператор $R_{i}$, удовлетворяющий условию (23). Покажем, что тогда решение $u_{i-1}$ системы $L_{i-1}(u)=0$ и соответствующий ему оператор $R_{i-1}$ можно найти по формулам

$$
\begin{aligned}
u_{i-1} & =\left(\frac{\partial}{\partial x}+B_{i}\right)\left(u_{i}\right)-R_{i}(f), \\
z_{i-1} & =\frac{\partial \widehat{H}_{i}}{\partial x}+B_{i-1} \widehat{H}_{i}-\widehat{H}_{i} B_{i}, \\
R_{i-1}(f) & =\int\left(\widehat{H}_{i} R_{i}(f)+z_{i-1} u_{i}\right) d y,
\end{aligned}
$$

выбирая в последней формуле константы интегрирования так, чтобы $Y_{i-1} R_{i-1}(f)=0$. 
Действительно, учитывая (20) и (21), нетрудно проверить, что

$$
\widehat{l}_{i-1} \circ\left(\frac{\partial}{\partial x}+B_{i}\right)=\left(\frac{\partial}{\partial x}+B_{i-1}\right) \circ \widehat{l}_{i}+z_{i-1} .
$$

В силу этой формулы и равенства (23) получаем

$$
\widehat{l}_{i-1}\left(\frac{\partial u_{i}}{\partial x}+B_{i} u_{i}\right)=\left(\frac{\partial}{\partial x}+B_{i-1}\right)\left(\frac{\partial R_{i}(f)}{\partial y}\right)+z_{i-1} u_{i} .
$$

Учитывая, что

$$
\left(\frac{\partial}{\partial x}+B_{i-1}\right)\left(\frac{\partial R_{i}(f)}{\partial y}\right)=\widehat{l}_{i-1}\left(R_{i}(f)\right)+\widehat{H}_{i} R_{i}(f),
$$

приходим к формуле

$$
\widehat{l}_{i-1}\left(\frac{\partial u_{i}}{\partial x}+B_{i} u_{i}-R_{i}(f)\right)=\widehat{H}_{i} R_{i}(f)+z_{i-1} u_{i} .
$$

Учитывая утверждение 4 и сопоставляя формулы (17) при $i$ и $i-1$, легко видеть, что столбцы матрицы $z_{i-1}$ лежат в ядре $Y_{i-1}$, а $Y_{i-1} \widehat{H}_{i} R_{i}(f)=Y_{i} R_{i}(f)=0$ в силу $(23)$. Поэтому

$$
\widehat{l}_{i-1}\left(u_{i-1}\right)=\widehat{H}_{i} R_{i}(f)+z_{i-1} u_{i} \in \operatorname{ker} Y_{i-1} \Rightarrow L_{i-1}\left(u_{i-1}\right)=Y_{i-1} \widehat{l}_{i-1}\left(u_{i-1}\right)=0 .
$$

Так как $Y_{m+1}=0$ и $L_{m}(u)=\left(Y_{m} u_{y}\right)_{x}+b Y_{m} u_{y}$, любой зависящий только от $x$ вектор $f(x)$ может быть взят в качестве решения системы $L_{m}(u)=0$. Обозначим $u_{m}=f(x)$. Нетрудно проверить, что соответствующий оператор $R_{m}$ находится по формуле $R_{m}=-\int \widehat{H}_{m+1} d y$. Стартуя с этих $u_{m}$ и $R_{m}$, по формулам $(24)$ найдем решение системы (1). Теорема доказана.

Из проведенного доказательства видно, что каскадный метод интегрирования Лапласа для систем уравнений вида (1) и проверка его применимости сводятся к выполнению следующей последовательности действий

1) Найти набор $n$ линейно независимых решений системы обыкновенных дифференциальных уравнений с параметром $w_{y}+a(x, y) w=0$ и, подставив эти решения в качестве столбцов в матрицу $\gamma(x, y)$, сделать в системе (1) замену переменных $u=\gamma \widetilde{u}$. После такого преобразования мы придем к системе вида (1) с нулевой матрицей $а$ и шаги 2)-4) алгоритма будем выполнять для этой преобразованной системы уравнений.

2) Найти $B_{i}$ по формулам (17), проверив тем самым их разрешимость, и выяснить выполнено ли условие $Y_{m+1}=0$ при некотором $m$. Матрицы $Y_{i}$ при этом удобно находить по формуле $Y_{i}=Y_{i-1} \widehat{H}_{i}$, где $\widehat{H}_{i}$ вычисляются по формуле (19).

3) Убедиться в разрешимости соотношений (14) - для этого достаточно, например, проверить, что при всех $i \leqslant m$ базисы ядер $Y_{i}$ могут быть составлены из векторов, не зависящих от $y$. (При практических вычислениях нам не требуется знать $a_{i}$.)

4) Если удалось выполнить шаги 2) и 3), то система (1) допускает построение решения вида (18) методом каскадного интегрирования Лапласа. В этом случае 
следует взять в качестве $u_{m}$ произвольный вектор $f(x)$ и в качестве $R_{m}$ - матрицу $\xi(x, y)$ такую, что $\xi_{y}=-\widehat{H}_{m+1}$ и $Y_{m} \xi=0$, а затем последовательно, начиная с $i=m-1$ и двигаясь в сторону уменьшения индекса $i$, найти $u_{i}$ и $R_{i}(f)$ по рекуррентным формулам (24), выбирая при этом константы интегрирования для $R_{i}(f)$ так, чтобы $Y_{i} R_{i}(f)=0$.

$5)$ Вернуться в исходную систему координат: $u=\gamma u_{0}$, где $\gamma-$ матрица, найденная нами в пункте 1), будет решением системы (1).

Проиллюстрируем этот алгоритм на примере системы

$$
\begin{aligned}
v_{x y}+\frac{v_{x}+v_{y}}{x+y}-8 \frac{\phi}{\psi^{2}} v-6 \frac{\psi}{\phi} w & =0, \\
w_{x y}+\frac{w_{x}+w_{y}}{x+y}+4 \frac{\phi}{\psi^{2}} v+6 \frac{\psi}{\phi} w & =0,
\end{aligned}
$$

где $\phi=\left(x^{3} y^{3}+3 x^{2} y^{2}-3 x y-1\right)^{2}, \psi=x^{4} y^{4}-4 x^{3} y^{3}-6 x^{2} y^{2}-4 x y+1$. При дальнейших вычислениях будем пользоваться тем, что $\phi_{x y} \phi-\phi_{x} \phi_{y}=6 \phi \psi$ и $\psi_{x} \psi_{y}-\psi_{x y} \psi=4 \phi$.

1) Сделав замену переменных $v=p /(x+y), w=q /(x+y)$, перейдем к системе

$$
\begin{aligned}
& p_{x y}-8 \frac{\phi}{\psi^{2}} p-6 \frac{\psi}{\phi} q=0, \\
& q_{x y}+4 \frac{\phi}{\psi^{2}} p+6 \frac{\psi}{\phi} q=0,
\end{aligned}
$$

которая не содержит первых производных по $x$.

2) Найдем последовательности $B_{i}, \widehat{H}_{i}$ и $Y_{i}$ для системы $(27)$ :

$$
\begin{gathered}
Y_{1}=H_{1}=\widehat{H}_{1}=\left(\begin{array}{cc}
8 \frac{\phi}{\psi^{2}} & 6 \frac{\psi}{\phi} \\
-4 \frac{\phi}{\psi^{2}} & -6 \frac{\psi}{\phi}
\end{array}\right), \\
B_{1}=\left(\begin{array}{cc}
\frac{\phi_{x}}{\phi}-2 \frac{\psi_{x}}{\psi} & 0 \\
0 & \frac{\psi_{x}}{\psi}-\frac{\phi_{x}}{\phi}
\end{array}\right), \quad \widehat{H}_{2}=\left(\begin{array}{cc}
-6 \frac{\psi}{\phi} & 6 \frac{\psi}{\phi} \\
-4 \frac{\phi}{\psi^{2}} & 4 \frac{\phi}{\psi^{2}}
\end{array}\right), \quad Y_{2}=\frac{24}{\psi}\left(\begin{array}{cc}
-3 & 3 \\
2 & -2
\end{array}\right), \\
B_{2}=\left(\begin{array}{cc}
-\frac{\psi_{x}}{\psi} & 0 \\
0 & -\frac{\psi_{x}}{\psi}
\end{array}\right), \quad \widehat{H}_{3}=\left(\begin{array}{cc}
-6 \frac{\psi}{\phi}-4 \frac{\phi}{\psi^{2}} & 6 \frac{\psi}{\phi} \\
-4 \frac{\phi}{\psi^{2}} & 0
\end{array}\right), \quad Y_{3}=\frac{144}{\phi}\left(\begin{array}{cc}
3 & -3 \\
-2 & 2
\end{array}\right), \\
\left.B_{3}=\left(\begin{array}{cc}
-\frac{\phi_{x}}{\phi} & 0 \\
0 & -\frac{\phi_{x}}{\phi}
\end{array}\right), \quad \begin{array}{cc}
-4 \frac{\phi}{\psi^{2}} & 6 \frac{\psi}{\phi} \\
-4 \frac{\phi}{\psi^{2}} & 6 \frac{\psi}{\phi}
\end{array}\right), \quad Y_{4}=0 .
\end{gathered}
$$

3) В качестве базисного вектора ядер $Y_{2}$ и $Y_{3}$ можно взять постоянный вектор $(1,1)$ и, следовательно, соотношения (14) для системы (27) разрешимы. Таким образом, все условия теоремы выполнены и мы можем использовать описанный выше алгоритм для построения решений системы (27). 
4) По формулам (24) построим последовательность $u_{i}$ и $R_{i}$ для системы (27):

$$
\begin{aligned}
& u_{3}=\left(\begin{array}{l}
f_{1}(x) \\
f_{2}(x)
\end{array}\right), \quad R_{3}=-\left(\begin{array}{ll}
\frac{\psi_{x}}{\psi} & \frac{\phi_{x}}{\phi} \\
\frac{\psi_{x}}{\psi} & \frac{\phi_{x}}{\phi}
\end{array}\right), \\
& u_{2}=\frac{d u_{3}}{d x}+\left(B_{3}-R_{3}\right) u_{3}, \quad R_{2}=\left(\begin{array}{cc}
2\left(\frac{\psi_{x}}{\psi}\right)_{x} & -\frac{\psi_{x x}}{\psi} \\
2\left(\frac{\psi_{x}}{\psi}\right)_{x} & -\frac{\psi_{x x}}{\psi}
\end{array}\right), \\
& u_{1}=\frac{\partial u_{2}}{\partial x}+B_{2} u_{2}-R_{2} u_{3}, \quad R_{1}=0, \\
& u_{0}=\left(\frac{\partial}{\partial x}+B_{1}\right)\left[\left(\frac{\partial}{\partial x}+B_{2}\right)\left(\frac{\partial}{\partial x}+B_{3}-R_{3}\right) u_{3}-R_{2} u_{3}\right] \text {. }
\end{aligned}
$$

5) Сделав обратную замену переменных, получаем, что

$$
\left(\begin{array}{c}
v \\
w
\end{array}\right)=\frac{u_{0}}{x+y}=\frac{1}{x+y}\left(\frac{\partial}{\partial x}+B_{1}\right)\left[\left(\frac{\partial}{\partial x}+B_{2}\right)\left(\frac{\partial}{\partial x}+B_{3}-R_{3}\right)-R_{2}\right]\left(\begin{array}{l}
f_{1}(x) \\
f_{2}(x)
\end{array}\right)
$$

является решением системы $(26)$ для любого вектора $\left(f_{1}(x), f_{2}(x)\right)$.

Во время подготовки настоящей публикации автору стало известно о работе [9], в которой предложена модификация каскадного метода интегрирования Лапласа для систем уравнений вида

$$
a_{i}(x, y) \frac{\partial u_{i}}{\partial x}+b_{i}(x, y) \frac{\partial u_{i}}{\partial y}=\sum_{j=1}^{n} c_{i j}(x, y) u_{j}, \quad i=1, \ldots, n,
$$

с попарно неколлинеарными векторами $\left(a_{i}, b_{i}\right)$. Не исключено, что после некоторых видоизменений этот алгоритм удастся применить и к системам вида (1), однако в нынешнем своем виде он для этого непригоден, так как в существенной мере опирается на свойство попарной неколлинеарности векторов $\left(a_{i}, b_{i}\right)$, которое не выполнено для $2 n$-компонентных систем, получающихся при записи системы (1) в виде (28).

Автор благодарен В. В. Соколову за вопросы, которые послужили первоначальным толчком к получению изложенного здесь результата, и за внимание к работе.

\section{СПИСОК ЦИТИРОВАННОЙ ЛИТЕРАТУРЫ}

[1] G. Darboux, Leçons sur la théorie générale des surfaces et les applications geometriques du calcul infinitesimal, v. 2, Gauthier-Villars, Paris, 1915.

[2] E. Goursat, Leçons sur l'intégration des équations aux dérivées partielles du second ordre à deux variables indépendantes, v. 1, Hermann, Paris, 1896; v. 2, Hermann, Paris, 1898.

[3] Ф. Трикоми, Лекиии по уравнениям в частных производных, ИЛ, М., 1957.

[4] А. В. Жибер, В. В. Соколов, "Точно интегрируемые уравнения лиувиллевского типа", УMH, 56:1 (2001), 63-106.

[5] А.В. Жибер, В.В. Соколов, С.Я. Старцев, "Нелинейные гиперболические системы уравнений лиувиллевского типа", Тихонов и современная математика: Функциональный анализ и дифференциалъные уравнения (Международная конференция, Москва, МГУ, 19-25 июля 2006 г.), Издательский отдел ф-та ВМиК МГУ им. М. В. Ломоносова, М., 2006, 305-306. 
[6] С. Я. Старцев, "Об инвариантах Лапласа систем гиперболических уравнений”, Комплексный анализ, дифференциальные уравнения, численные методы и приложения, 3, Ин-т математики с ВЦ РАН, Уфа, 1996, 144-154.

[7] А.В. Жибер, С.Я. Старцев, "Интегралы, решения и существование преобразований Лапласа линейной гиперболической системы уравнений”, Матем. заметки, 74:6 (2003), 848-857.

[8] А. М. Гурьева, А. В. Жибер, “Инварианты Лапласа двумеризованных открытых цепочек Тоды", ТМФ, 138:3 (2004), 401-421.

[9] S.P. Tsarev, Generalized Laplace transformations and integration of hyperbolic systems of linear partial differential equations, arXiv: cs/0501030.

С. Я. Старцев

Институт математики

с вычислительным центром РАН

E-mail: startsev@anrb.ru
Поступило

29.04 .2005

Исправленный вариант

19.04.2007 